\title{
Heart Sound Analysis for Discrimination of VSD
}

\section{Shuping Sun ${ }^{1, a}$ Zhongwei Jiang ${ }^{1, b}$ Haibin Wang ${ }^{2, c}$ Ting Tao ${ }^{2, d}$}

${ }^{1}$ Department of Mechanical Engineering, Faculty of Engineering, Yamaguchi University, Japan

${ }^{2}$ School of Electrical and Information Engineering, Xihua University, China

ap006wc@yamaguchi-u.ac.jp, bJiang@yamaguchi-u.ac.jp ddwang@xhu.edu.cn, dandyting51@sina.com

Keywords: Ventricular septal defect (VSD), Heart sounds (HSs), Characteristic waveform in time domain $\left(\mathrm{W}_{t}\right)$, Characteristic Waveform in frequency domain $\left(\mathrm{W}_{f}\right)$, common region

\begin{abstract}
A ventricular septal defect (VSD) is the most common congenital heart disease, which can be cured with a high probability if it is detected in an early stage. In our previous researches on heart sounds (HSs) analysis, the detection methods of heart disease using the cardiac sound characteristic waveforms in time domain or in frequency domain were proposed, and have been succeed in discriminating several heart murmurs. In this paper, we are going to apply these methods to detect VSD. Based on analysis results, a new approach by using the feature parameters both in time domain and in frequency domain is proposed to achieve higher discrimination rates.
\end{abstract}

\section{Introduction}

A VSD is one of the most common congenital heart disease, accounting for $0.24 \%$ of newborn babies [1,2], and it can be cured with a high probability if this disease is detected in an early stage. Recently, with the high development of computer technique and digital signal processing technology [3,6], more and more researches are concerning on the HSs analysis. Through comparative analysis, the simple and effective methods of our previous studies $[4,6]$ were proposed to detect heart murmurs with higher classification accuracy. In this study, we are going to use our previous method to detect VSD from normal cases. According to experimental results, a new approach is proposed to achieve higher discrimination rates. The rest of the paper is organized as follows. Section2 introduces HSs acquisition and preprocessing. Section3 proposes HSs analysis method. Section 4 presents discrimination analysis. Finally, the conclusions are summarized in Section5.

\section{HSs Acquisition and preprocessing}

Auscultation denotes the act of analyzing sounds in the body that is produced in response to mechanical vibrations generated in the organs. Therefore, for different heart murmurs, we should analyze HSs collected from different auscultation areas, while for VSD cases, it is reported that the HSs collected from tricuspid area can supply more important information [8]. In this study, analyzed HSs were collected from tricuspid area by the HSs acquisition system, meanwhile, sampling frequency Fs were set as $44.1 \mathrm{kHz}$. The basic HSs consist of two primary components which are often described as the first heart sound (S1) and second heart sound (S2). To analyze the useful information, wavelet decomposition (WD) is used as pre-processing for cancellation of the unwanted frequency components over $700 \mathrm{~Hz}$ and below $20 \mathrm{~Hz}$. Daubechies type wavelet DB10 is used as a mother wavelet. Finally, the filtered signals, $x(t)$ with $21.5-689 \mathrm{~Hz}$ is gained.

\section{Cardiac sound analysis}

In this section, firstly, the diagnostic features, $[\mathrm{T} 11, \mathrm{~T} 12]$ from characteristic waveform $\left(\mathrm{W}_{t}\right)$ in time domain as detecting character of HSs are extracted. And then to experimental analysis, the diagnostic features $[\mathrm{Fg}, \mathrm{Fw}]$ extracted from the frequency characteristic waveform $\left(\mathrm{W}_{f}\right)$ are proposed to detect VSD. By analyzing the distribution of [T11,T12] and [Fg,Fw] for VSD and normal HSs. Finally, a new approach is proposed for detecting VSD and normal cases. 


\subsection{Time domain analysis}

In our previous studies, time characteristic waveform analysis method [4] could realize detection of several heart diseases. So this method is used to analyze performance evaluation for discriminating VSD HSs and normal HSs, and the detailed is in following.

\subsubsection{Characteristic waveform( $\left(\mathbf{W}_{\mathbf{t}}\right)$ extraction}

Consider a data series $x(t), t=1,2, \ldots, N$, by WD for HSs, where $N$ denotes the number of data. Then the $\mathrm{W}_{t}$ based on the Viola integral method is given by

$$
\mathrm{w}_{\mathrm{z}}(\delta)=\frac{1}{2 \delta} \sum_{\mathrm{n}=\mathrm{t}-\delta+1}^{\mathrm{t}+\delta}\left[\mathrm{x}(\mathrm{n})-\sum_{\mathrm{n}=\mathrm{t}-\delta+1}^{\mathrm{t}+S} \mathrm{x}(\mathrm{n}) / 2 \delta\right]^{2}
$$

At last, the normalization is applied by setting the amplitude of $\mathrm{W}_{t}$ within 1.0. Since many experiments show that the duration of $\mathrm{S} 1$ or $\mathrm{S} 2$ is over than 0.06 second [4,8], we set $\delta=0.03 \times \mathrm{Fs}=1323$. As an example, Fig. 1 plots $x(\mathrm{t})$ daubed with gray and it's $\mathrm{W}_{t}$. Fig. 1 (a) shows the case of normal sound and Fig.1(b) is VSD sound. As for the normal sound, $\mathrm{W}_{t}$ seems to have longer time interval between two abutted S1 and between S1 and S2 than VSD.

\subsubsection{Diagnostic features definition and representation}

As mentioned above, a concept for defining the diagnostic parameters is described in Fig.1(a) and (b). A threshold value (Thv1) is selected first at a suitable value, the time intersection between the crossed points of the $\mathrm{W}_{t}$ on the Thv1 line are defined by a( $\left.\mathrm{S} 1_{i}\right), \mathrm{b}\left(\mathrm{S} 1_{i}\right), \mathrm{a}\left(\mathrm{S} 2_{i}\right)$ and $\mathrm{b}\left(\mathrm{S} 2_{i}\right)(i=1,2, ., N)$ in a sequential order as shown in Fig. 1 . The center of gravity, especially denoted $\mathrm{G}_{t}\left(\mathrm{~S}_{i}\right)$ and $\mathrm{G}_{t}\left(\mathrm{~S} 2_{i}\right)$ as shown in Fig.1(a) and (b), the time index of $\mathrm{G}_{t}\left(\mathrm{~S}_{i}\right)$ and $\mathrm{G}_{t}\left(\mathrm{~S} 2_{i}\right)$ are gained by

$$
\mathrm{G}_{\mathrm{t}}\left(\mathrm{Sk}_{\mathrm{j}}\right)=\Sigma_{\mathrm{n}=\mathrm{a}\left(\mathrm{sk} \mathrm{k}_{\mathrm{i}}\right)}^{\mathrm{b}\left(\mathrm{Sk}_{\mathrm{j}}\right)} \mathrm{n} \times \mathrm{W}_{\mathrm{t}}(\mathrm{n})^{2} / \Sigma_{\mathrm{n}=\mathrm{a}\left(\mathrm{sk} \mathrm{k}_{\mathrm{i}}\right)}^{\mathrm{b}\left(\mathrm{Sk}_{\mathrm{j}}\right)} W_{\mathrm{t}}(\mathrm{n})^{2}, \mathrm{k}=1,2 ; \mathrm{i}=1,2, \ldots, \mathrm{N} \text {. }
$$

$\mathrm{T} 11_{i}$ is the time interval between $\mathrm{G}_{t}\left(\mathrm{~S}_{i}\right)$ and $\mathrm{G}_{t}\left(\mathrm{~S} 1_{i+1}\right), \mathrm{T} 12_{i}$ is the time interval between $\mathrm{G}_{t}\left(\mathrm{~S}_{i}\right)$ and $\mathrm{G}_{t}\left(\mathrm{~S} 2_{i}\right)$. To make the parameters $\left[\mathrm{T} 11_{i}, \mathrm{~T} 12_{i}\right]$ visually, a two-dimensional plot, scatter gram, on $\left[\mathrm{T} 11_{i}\right.$, $\left.\mathrm{T} 12_{i}\right]$ is introduced as shown in Fig.1(c).
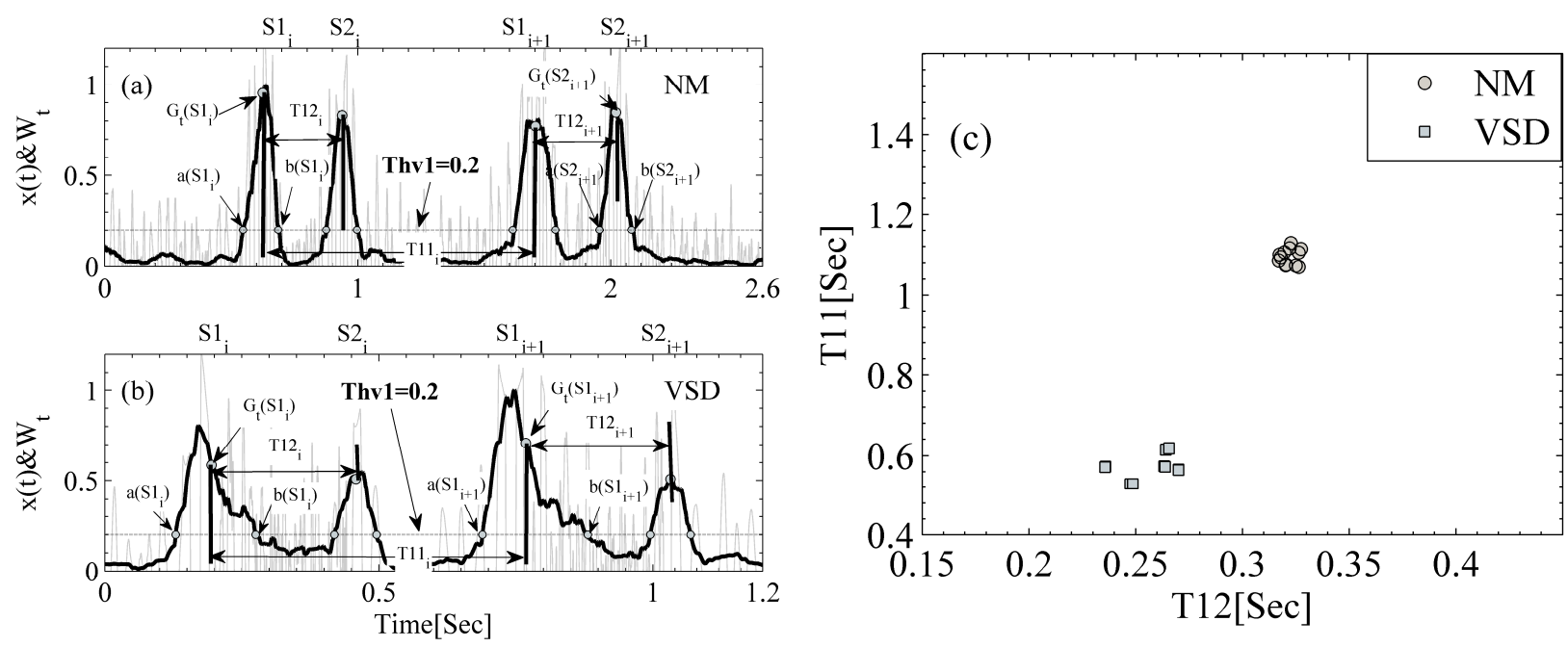

Fig.1. Concept for defining the diagnostic features [T11, T12] from Wt and [T11, T12]'s representation by scatter diagram. (a) Normal sound case, (b) abnormal case of VSD and (c)

$[\mathrm{T} 11, \mathrm{~T} 12]$ distribution for NM and VSD cases.

\subsubsection{Effects of Thv1 on [T11,T12]}

By many experimental analysis, as for normal cases, when Thv1 is set as the interval $[0.2,0.5]$, $[\mathrm{T} 11, \mathrm{~T} 12]$ is almost not sensitive with Thv1.While as for VSD cases, when Thv1 is set as [0.2,0.4], there is a little influence on [T11,T12], which can be negligible. 


\subsubsection{Experimental results and discussions}

To validate the proposed method, in this paper, the used data set with 468 sound samples that consisted of 242 normal and 226 VSD cases. The normal cases were from 23 health students in university, the VSD cases were from 17 patients in hospital.

\subsubsection{Case of normal HS}

According to the distribution of [T11,T12], generally, normal HSs could be divided into three types. Fig.2(a),(b) and (c) show the three typical examples collected from a health female of age 25 with weight $61 \mathrm{~kg}$ (NM1), a healthy male of age 26 with weight $72 \mathrm{~kg}$ (NM2), a male of age 23 with weight $68 \mathrm{~kg}$ (NM3), respectively. Furthermore, the data set of the diagnostic features [T11,T12] are plotted in Fig.2(g), NM1 is marked by $(\square)$, NM2 $(\nabla)$, and NM3( $)$.
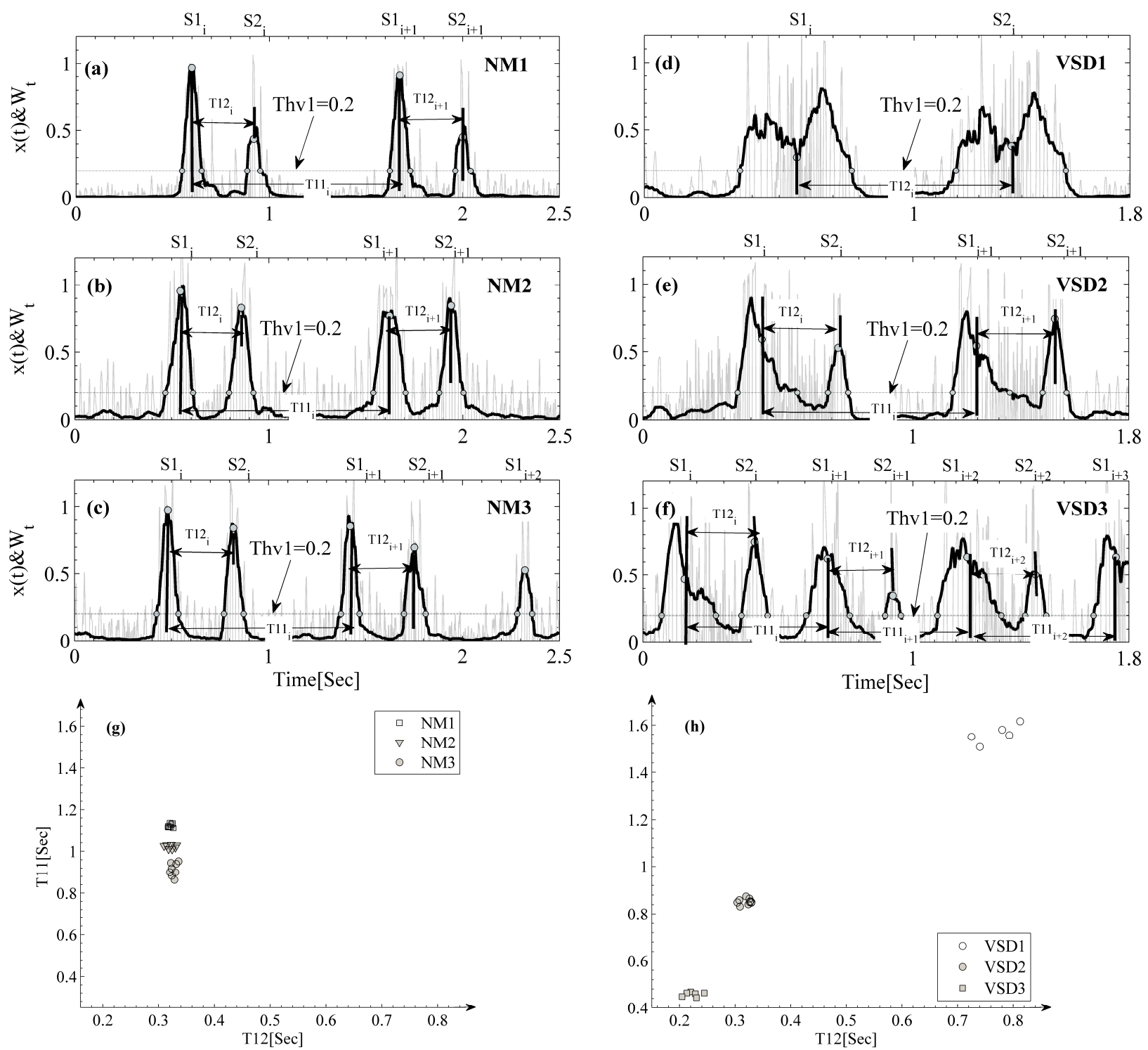

Fig.2. Plots of the Wt curves and [T11,T12] extraction and the graphic representations of three kinds of normal heart sound signals(NM1,NM2,NM3), and three kinds of VSD heart sounds(VSD1,VSD2,VSD3).

\subsubsection{Case of VSD HS}

However, for VSD cases, according to the strength of noise and the heart beat, VSD case generally can be divided into three types. The first type is that S1 and S2 can not be distinguished due to stronger noise which causes [T11,T12] are far bigger than NM case, just as a VSD1 case collected from a male with VSD of age 12 and $32 \mathrm{~kg}$ (Fig.2(d)). The second is that the noise almost not affect the [T11,T12], just as VSD2 from female of age 7 with weight $30 \mathrm{~kg}$ (Fig.2(e)). The third is the 
case with higher heart beat and heart noise, named VSD3 from a female of age 4 with weight $16 \mathrm{~kg}$, the distribution of [T11,T12] are a far smaller than NM cases (Fig.2(f)). Furthermore, the data set of

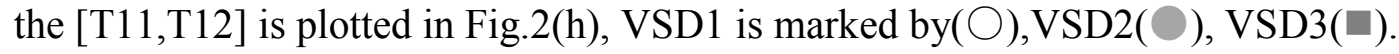

\subsubsection{Summary}

Analysis results show that there is the common region between VSD cases and NM cases, which includes $32.6 \%$ of NM cases and $29.1 \%$ of VSD cases, so it's impossible to distinguish VSD cases from NM cases. Next, frequency analysis method is proposed to detect normal and VSD cases.

\section{2 frequency analysis method}

The envelope curve method in frequency domain has been proved to recognize several heart murmurs in our previous studies [6].Based on this point, a new characteristic waveform curve in frequency domain $\left(\mathrm{W}_{f}\right)$ is proposed to detect VSD. Consider a data series $x(\mathrm{t}), t=1,2, \ldots, N$, where, $N$ denotes the number of data. Then $\mathrm{W}_{f}(f=1,2, . . N)$ based on the Viola integral method is given by

$$
w_{f}(\delta)=\frac{1}{2 \delta} \sum_{k=f-\delta+1}^{f+\delta}\left|\left(\sum_{n=0}^{N-1} x(\boldsymbol{t}) e^{-j \frac{2 \pi}{N} k t}\right)\right| \text {. }
$$

By many experiments, $\delta$ is set as 8 . At last, the normalization is applied by setting the amplitude of $\mathrm{W}_{f}$ within 1.0. As an example, frequency distribution daubed with gray and $\mathrm{W}_{f}$ are plotted in Fig.3. Fig.3(a) shows a normal case and Fig.3(b) is a VSD case. $\mathrm{W}_{f}$ of the normal sounds, which has the lower density frequency component focused on a narrower region compared with VSD cases.

\subsubsection{Features extraction and representation}

Two diagnostic features, Fg and Fw are defined, which correspond to the frequency index of the center of $\mathrm{W}_{f}$ during interval $[0,(\mathrm{~N}-1) / 2]$ and the frequency width of $\mathrm{W}_{f}$ on a Thv2 as shown in Fig.3(a) and (b).The representation of [Fg,Fw] is introduced in Fig.3(c). Here, Fg is gained by

$$
F g=\Sigma_{k=0}^{(N-1) / 2} k W_{f}(k) / \Sigma_{k=0}^{(N-1) / 2} W_{f}(k) .
$$
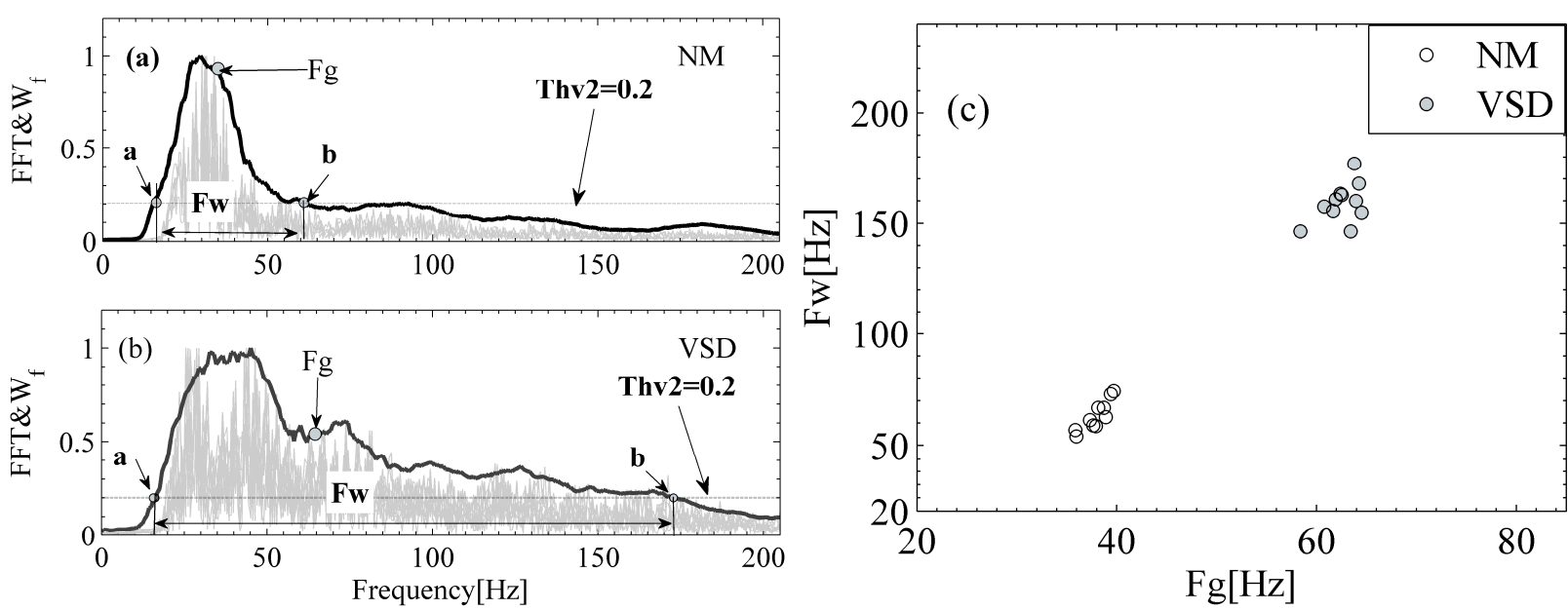

Fig.3. Concept for defining the diagnostic features [Fg,Fw] from Wf curve and [Fg,Fw]'s representation by scatter diagram. (a) Normal sound case, (b) abnormal case of VSD and (c)

$[\mathrm{Fg}, \mathrm{Fw}]$ distribution for NM and VSD cases.

By many experimental analysis, when Thv2 is selected in the interval [0.1,0.2]. Generally, there are greater differences between NM cases and VSD cases. In this study, the Thv2 is set at 0.2.

\subsubsection{Experimental results and discussions}

Character of $\mathrm{W}_{f}$ as shown in Fig.4(a-f). Fig.4(g) and (h) show the data set of the $[\mathrm{Fg}, \mathrm{Fw}]$. 


\subsubsection{Summary}

By analysis, the common region is extracted, which includes $38.4 \%$ of $\mathrm{NM}$ cases and $20.1 \%$ of VSD cases. So it's also impossible to distinguish VSD cases from NM cases using [Fg,Fw]. But by analyzing common region, the common region mainly consists of NM3 and VSD2 in time domain, while the common region in frequency domain mainly consists of NM1 and VSD3. So using $[\mathrm{T} 11, \mathrm{~T} 12] \&[\mathrm{Fg}, \mathrm{Fw}]$ as new diagnostic features might discriminate VSD from NM cases.

\section{Discrimination analysis using $[\mathrm{T} 11, \mathrm{~T} 12] \&[\mathrm{Fg}, \mathrm{Fw}]$}

The distributions of [Fg, Fw] for the NM and VSD cases in [T11,T12] common region are analyzed, the experimental results are showed in Fig.5. Fig.5(a) shows the [T11,T12] in common region,
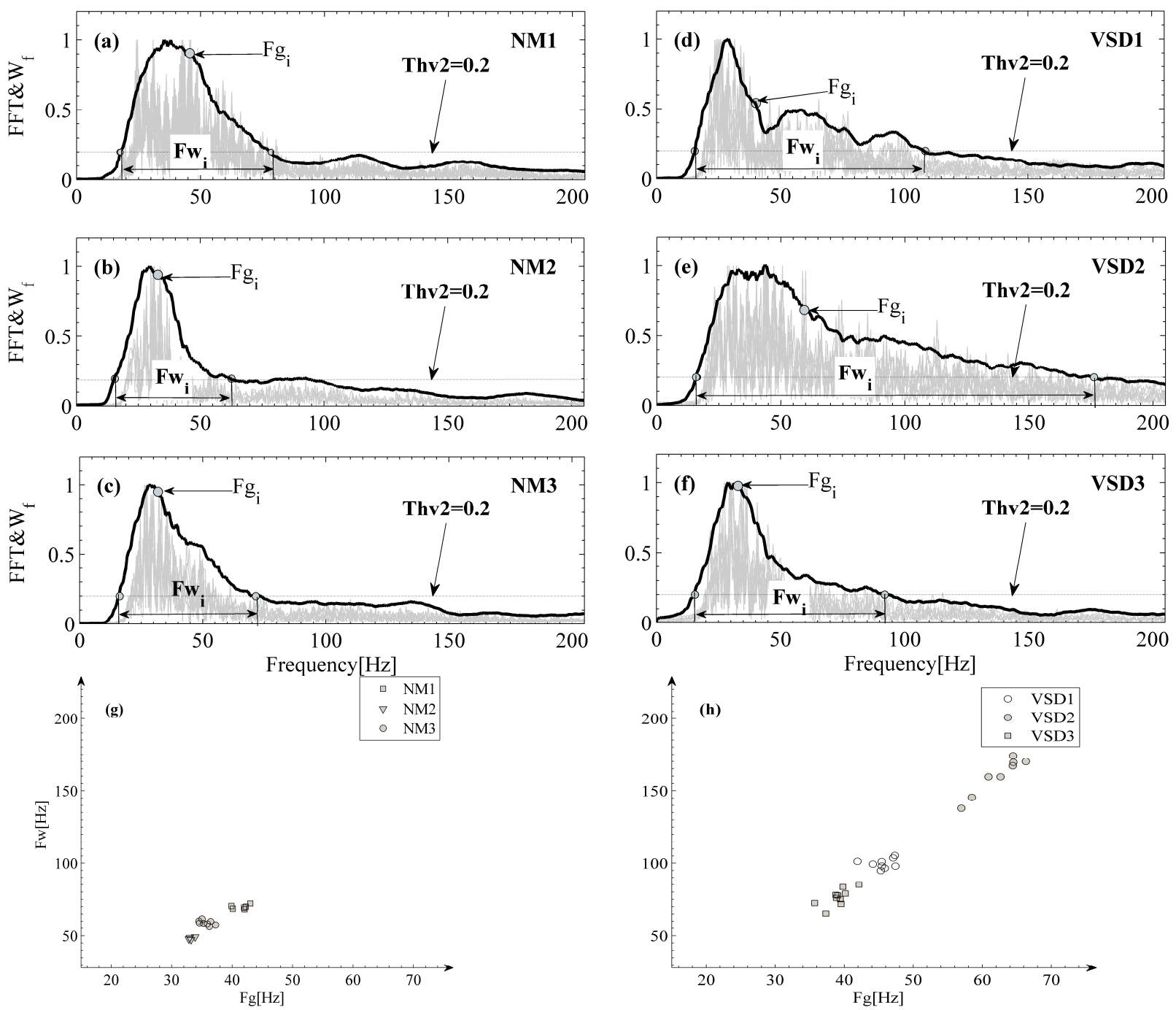

Fig.4. Plots of the Wf curves and [Fg,Fw] extraction and the graphic representations of three kinds of normal heart sound signals(NM1,NM2,NM3), and three kinds of VSD heart sounds(VSD1,VSD2,VSD3)

which include $32.6 \%$ of total NM cases denoted NMc , and $20.1 \%$ of total VSD cases denoted VSDc and Fig.5(b) is the distributions of NMc and VSDc in [Fg,Fw] domain, in [Fg,Fw] common region, $3.4 \%$ of NMc and $8.5 \%$ of VSDc are included. So the higher discrimination rates for NM cases denoted DRN and VSD cases (DRV) are gained by

$$
D R N=1-32.6 \% \times 3.4 \%=98.89 \% ; \quad D R V=1-29.1 \% \times 8.5 \%=97.53 \%
$$



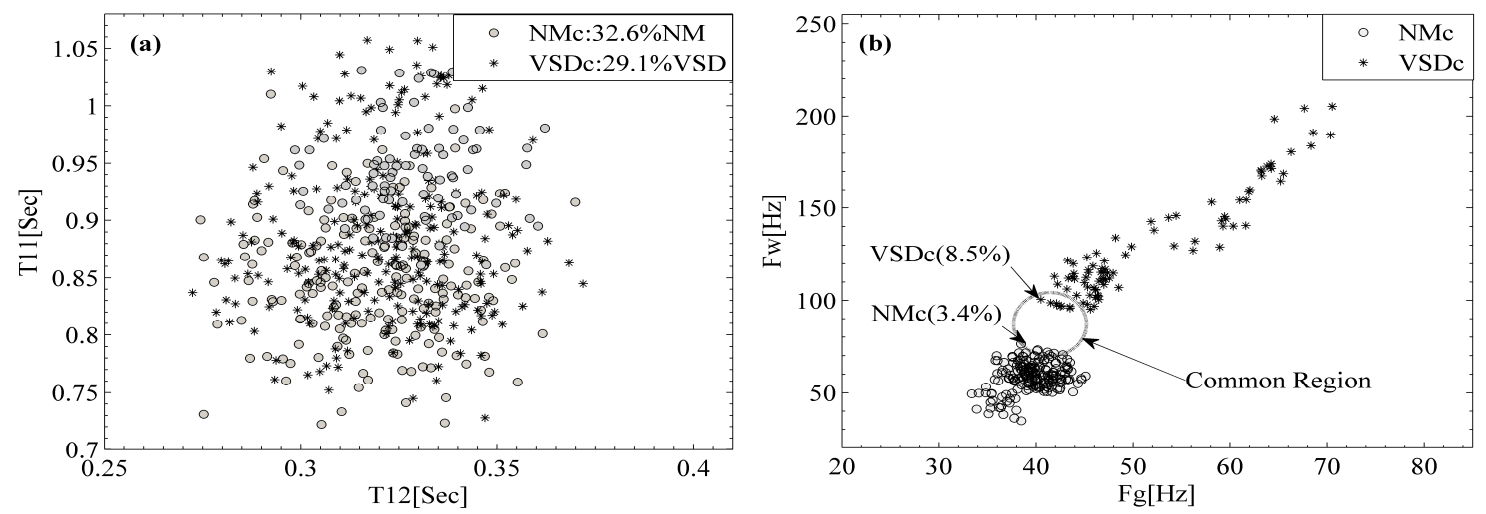

Fig.5. (a) is the graphic representations of the data set [T11,T12] for VSD and NM cases in common region and (b) shows $[\mathrm{Fg}, \mathrm{Fw}]$ representation corresponding to Fig.5(a) heart sounds

\section{Conclusion}

In this study, the character waveform method for discriminating VSD and NM cases was presented in detail. At first, the WD was used for retaining the frequency components between the interval $[20,700] \mathrm{Hz}$. Next, two diagnostic features [T11,T12] extracted from $\mathrm{W}_{t}$ were defined for detecting VSD, A case study on the normal and VSD sounds was demonstrated to validate the usefulness and efficiency of $\mathrm{W}_{t}$. Based on analysis results for discriminating NM and VSD cases, in frequency domain, $[\mathrm{Fg}, \mathrm{Fw}]$ extracted from $\mathrm{W}_{f}$ were defined as diagnostic features for detecting VSD cases. By analyzing the distribution of [T11,T12] and [Fg,Fw], a new approach using [T11,T12]\&[Fg,Fw] as diagnostic features for detecting VSD and normal cases was proposed. Experimental results showed discrimination rate are $\mathrm{DRN}=98.89 \%, \mathrm{DRV}=97.53 \%$, respectively.

\section{Reference}

[1] Ambumani P, Kuruchi Srinivasan. Ventricular Septal Defect, General Concepts. eMedicine.com. URL: http://www.emedicine.com/ped/topic2402.htm. December 5, 2005

[2] Eidem BW. Ventricular Septal Defect, Muscular. eMedicine.com. URL: http://www.emedicine.com/ped/topic2543.htm. Accessed on April 13, 2006.

[3] M. El-Segaier, O. Lilja, S. Lukkarinen, L. Sörnmo, R. Sepponen and E. Pesonen, Computer-based detection and analysis of heart sound and murmur, Annals of Biomedical Engineering 33 (7) (2005), pp. 937-942.

[4] Zhongwei Jiang,Samjin Choi, A cardiac sound characteristic waveform method for in-home heart disorder monitoring with electric stethoscope. Expert Systems with Applications 31(2006) 286-298.

[5] Zümray Dokur, Tamer Ölmez, Heart sound classification using wavelet transform and incremental self-organizing map, Digital Signal Processing, Volume 18, Issue 6, November 2008, Pages 951-959

[6] Samjin Choi,Zhongwei Jiang, Cardiac sound murmurs classification with auto regressive spectral analysis and multi-support vector machine technique. Computers in Biology and Medicine 40 (2010) 8-20

[7] Vapnik, V. (1989). Statistical Learning Theory. New York: Wiley.

[8] D. Kumar, P. Carvalho, M. Antunes, P. Gil, J. Henriques, L.Eugenio, A new algorithm for detection of S1 and S2 heart sounds, in: ICASSP 2006 Proceedings, vol. 2, Acoust. Speech Signal Process. (2006). 